\title{
ABSOLUTELY CONTINUOUS FUNCTIONS ON IDEMPOTENT SEMIGROUPS IN THE LOCALLY CONVEX SETTING
}

BY

\section{A. KATSARAS}

\begin{abstract}
Let $E$ be a locally convex space and let $T$ be a semigroup of semicharacters on an idempotent semigroup. It is shown that there exists an isomorphism between the space of $E$-valued functions on $T$ and the space of all $E$-valued finitely additive measures on a certain algebra of sets. The space of all $E$-valued functions on $T$ which are absolutely continuous with respect to a positive definite function $F$ is identified with the space of all $E$-valued measures which are absolutely continuous with respect to the measure $m_{F}$ corresponding to $F$. Finally a representation is given for the operators on the set of all $E$-valued finitely additive measures on an algebra of sets which are absolutely continuous with respect to a positive measure.
\end{abstract}

Introduction. Functions of bounded variation and absolutely continuous functions have been studied by several authors including [1], [2] , [5], [6], [7], [8] and [9]. In [6] a representation is obtained of the linear functionals on $A C(I)$ which are continuous in the bounded-variation norm in terms of the $v$ integral. In [8] and [9] the concepts of bounded variation and absolutely continuous are developed on idempotent semigroups. In [1] and [2] the results of [8] and [9] are used to extend the $v$-integral characterization of functionals in [6] to a $v$-integral characterization of normed vector space-valued operators on normed vector space-valued absolutely continuous functions on an idempotent semigroup.

In this paper we study the space of functions from a semigroup $T$ of semicharacters on an idempotent semigroup $S$ into a locally convex space $E$. We identify $E$-valued functions on $T$ with $E$-valued finitely additive measures on a certain algebra of subsets of $S$, and then represent operators on this set of finitely additive measures. To this end we adopt the notation and development in [8] and [9].

Received by the editors May 15, 1973 and, in revised form, March 10, 1974. AMS (MOS) subject classifications (1970). Primary 46G10, 46E40; Secondary 28A25, 28A45.

Key words and phrases. Bounded variation, absolutely continuous, v-integral, locally convex space, continuous seminorm, semicharacter, semigroup, positive-definite, polygonal function, simple function, finitely additive measure. 
1. Definitions. Let $S$ be an abelian idempotent semigroup and let $T$ be a semigroup of semicharacters on $S$ containing the identity semicharacter. Let $A$ denote the algebra of subsets of $S$ generated by the sets $J_{f}, f \in T$ (see [8]). Assume that $E$ is a real locally convex Hausdorff space and let $\{p: p \in I\}$ be a generating family of continuous seminorms on $E$ which is directed, i.e., given $p_{1}$, $p_{2}$ in $I$ there exists $p \in I$ such that $p \geqslant p_{1}, p_{2}$ (pointwise). For each $p \in I$, let $B V(T, E)_{p}$ denote the space of all functions $G: T \rightarrow E$ for which

$$
\|G\|_{B V, p}=\sup \sum_{\tau} p(L(Z, \tau))<\infty
$$

where the supremum is taken over the collection of all finite subsets $Z$ of $T$. Set

$$
B V(T, E)=\bigcap_{p \in I} B V(T, E)_{p} .
$$

Clearly $B V(T, E)$ is a real vector space. Let $\theta$ denote the locally convex topology on $B V(T, E)$ generated by the family of seminorms $\left\{\|\cdot\|_{B V, p}: p \in I\right\}$. If $G$ is a real-valued function on $T$ and $x \in E$, then $G x$ is defined on $T$ by $(G x)(f)=$ $G(f) x$. Clearly

$$
\{G x: G \in B V(T), x \in E\} \subset B V(T, E) .
$$

Let now $F$ be positive definite. We denote by $A C(T, E, F)$ the $\theta$-closure of the space spanned by $G x, x \in E$ and $G$ in the space $A C(T, E)$ of all real functions on $T$ which are absolutely continuous with respect to $F$. If $G \in A C(T, E, F)$, we say that $G$ is absolutely continuous with respect to $F$ and write $G \ll F$.

2. Finitely additive $E$-valued measures on $A$. Let $p \in I$. We denote by $M_{p}(A, E)$ the collection of all finitely additive $E$-valued measures $m$ on $A$ for which sup $\sum_{j=1}^{n} p\left(m\left(B_{j}\right)\right)=\|m\|_{p}<\infty$ where the supremum is taken over all finite partitions $\left\{B_{j}\right\}$ of $S$ into sets in $A$. Since every element of $A$ is a disjoint union of sets of $B$-type, it suffices to take the supremum over partitions of $S$ into sets of $B$-type. Let

$$
M(A, E)=\bigcap_{p \in I} M_{p}(A, E) .
$$

We denote by $\omega$ the locally convex topology on $M(A, E)$ generated by the seminorms $\left\{\|\cdot\|_{p}: p \in I\right\}$.

Let now $\mu$ be a real-valued finitely additive set function on $A$ and let $m$ be an $E$-valued finitely additive measure on $A$. Let $\Sigma$ denote the algebra of subsets of $S \times S$ generated by the sets $B_{1} \times B_{2}, B_{1}$ and $B_{2}$ in $A$. Every element of $\Sigma$ can be written as a finite disjoint union of sets of the form $B_{1} \times B_{2}$ with $B_{1}, B_{2}$ in $A$. It is not hard to show that there exists a unique $E$-valued finitely additive 
measure $\mu \times m$ on $\Sigma$ such that $\mu \times m\left(B_{1} \times B_{2}\right)=\mu\left(B_{1}\right) m\left(B_{2}\right)$ for all $B_{1}, B_{2}$ in $A$. Let $\pi: S \times S \rightarrow S$ be defined by $\pi(s, t)=$ st. If $B \in A$, then $\pi^{-1}(B) \in \Sigma$. We define the convolution $\mu * m$ on $A$ by $\mu * m(B)=\mu \times m\left(\pi^{-1}(B)\right)$. It follows easily that $\mu * m$ is finitely additive. Moreover, we have the following:

Lemma 1. If $\mu$ is bounded and $m \in M_{p}(A, E)$, then $\mu * m \in M_{p}(A, E)$.

Proof. Let $Z=\left\{f_{1}, \cdots, f_{n}\right\} \subset T$ and $B_{\tau}=B(Z, \tau)$. By [8] we have $\pi^{-1}\left(B_{\tau}\right)=\bigcup\left\{B_{\tau_{1}} \times B_{\tau_{2}}: \tau_{1}, \tau_{2}\right.$ in $\left.T_{n}, \tau_{1} \wedge \tau_{2}=\tau\right\}$.

Hence

$$
p\left(\mu * m\left(B_{\tau}\right)\right) \leqslant \sum_{\tau_{1} \wedge \tau_{2}=\tau}\left|\mu\left(B_{\tau_{1}}\right)\right| p\left(m\left(B_{\tau_{2}}\right)\right)
$$

Thus

$$
\begin{aligned}
\sum_{\tau \in T_{n}} p\left(\mu * m\left(B_{\tau}\right)\right) & \leqslant \sum_{\tau_{1}}\left|\mu\left(B_{\tau_{1}}\right)\right| \sum_{\tau_{2}} p\left(m\left(B_{\tau_{2}}\right)\right) \\
& \leqslant\|m\|_{p} \sum_{\tau_{1}}\left|\mu\left(B_{\tau_{1}}\right)\right| \leqslant\|m\|_{p}\|\mu\| .
\end{aligned}
$$

This implies that $\|\mu * m\|_{p} \leqslant\|\mu\|\|m\|_{p}$.

For $\mu$ a real-valued finitely additive measure on $A$ and for $x$ in $E$ we define $\mu x$ on $A$ by $(\mu x)(B)=\mu(B) x$. If $\mu$ is bounded, then $\|\mu x\|_{p}=\|\mu\| p(x)$ for each $p$ in $I$. Let now $m$ be a nonnegative finitely additive measure on $A$. A real-valued bounded finitely additive measure $\mu$ on $A$ is said to be absolutely continuous with respect to $m$, and write $\mu \ll m$, if for every $\epsilon>0$ there exists $\delta>0$ such that $m(B)<\delta$ implies $|\mu|(B)<\epsilon$. An $E$-valued measure $\lambda$ on $A$ is said to be absolutely continuous with respect to $m$, and we write $\lambda \ll m$, if $\lambda$ is in the $\omega$ closure of the subspace of $M(A, E)$ spanned by $\mu x$, where $x \in E$ and $\mu$ a bounded real-valued measure on $A$ with $\mu \ll m$.

3. Relationship between $E$-valued functions on $T$ and finitely additive measures on $A$. For each finitely additive $E$-valued measure $m$ on $A$, we define $\hat{m}: T \rightarrow E, \hat{m}(f)=m\left(A_{f}\right)=\int f d m$. This gives us a map from the set of all finitely additive measures on $A$ into the set of all functions from $T$ into $E$. The following theorem gives the properties of this map.

THEOREM 1. The map $m \rightarrow \hat{m}$, from the space of all finitely additive $E$ valued measures on $A$ into the space of all $E$-valued functions on $T$, is linear oneto-one and onto. Moreover the following hold:

(a) $m \in M_{p}(B, E)$ iff $\hat{m} \in B V(T, E)_{p}$. 
(b) $\|m\|_{p}=\|\hat{m}\|_{B V, p}$.

(c) $m \ll a$ iff $\hat{m} \ll \hat{a}$.

(d) $\widehat{\mu x}=\hat{\mu} x$ for every real measure $\mu$ on $A$ and every $x \in E$.

(e) $\widehat{\mu * m}=\hat{\mu} \hat{m}$.

(f) If $h:(M(A, E), \omega) \rightarrow(B V(T, E), \theta), h(m)=\hat{m}$, then $h$ is a topological isomorphism.

Proof. The proof that the map $m \rightarrow \hat{m}$ is linear, one-to-one and onto is similar to the one in the scalar case (see [8]). Since $m(B(Z, \tau))=L(Z, \tau) \hat{m}$ for each set $B(Z, \tau)$ of $B$-type, it follows easily that $\|m\|_{p}=\|\hat{m}\|_{B V, p}$ and hence $m \in M_{p}(B, E)$ iff $\hat{m} \in B V(T, E)_{p}$. Also, from the $\widehat{\mu x}(f)=(\mu x)\left(A_{f}\right)=\mu\left(A_{f}\right) x=$ $\hat{\mu}(f) x$, it follows that $\widehat{\mu x}=\hat{\mu x}$ for each real-valued measure $\mu$ on $A$ and each $x \in E$.

Assume next that $a$ is a positive measure on $A$ and $m$ an $E$-valued measure on $A$. Suppose that $m \ll a$ and let $p \in I$ and $\epsilon>0$. There are bounded realvalued measures $\mu_{1}, \cdots, \mu_{n}$ on $A$, with $\mu_{i} \ll a$, and $x_{1}, \cdots, x_{n} \in E$ such that $\left\|\Sigma \mu_{i} x_{i}-m\right\|_{p}<\epsilon$. Thus $\left\|\Sigma \hat{\mu}_{i} x_{i}-\hat{m}\right\|_{B V, p}=\left\|\Sigma \mu_{i} x_{i}-m\right\|_{p}<\epsilon$. Since $\hat{\mu}_{i} \ll \hat{a}$, this shows that $\hat{m} \ll \hat{a}$. The proof of the converse is similar. Finally, from

$$
\begin{aligned}
\widehat{\mu * m}(f) & =\mu * m\left(A_{f}\right)=\mu \times m\left(\pi^{-1}\left(A_{f}\right)\right)=\mu \times m\left(A_{f} \times A_{f}\right) \\
& =\mu\left(A_{f}\right) m\left(A_{f}\right)=\hat{\mu}(f) \hat{m}(f),
\end{aligned}
$$

it follows that $\widehat{\mu * m}=\hat{\mu} \hat{m}$.

For $G$ a real-valued or $E$-valued function on $T$ we denote by $m_{G}$ the measure on $A$ such that $\hat{m}_{G}=G$.

Let now $F$ be a positive definite function on $T$. For each $E$-valued simple function $s$ we define $v_{s}: A \rightarrow E$ by

$$
v_{s}(B)=\int_{B} s d m_{F} .
$$

Clearly $v_{s} \ll m_{F}$. By Theorem 1 we have $\hat{v}_{s} \ll F$. The function $\hat{v}_{s}=p_{s}$ is called a polygonal function.

THEOREM 2. If $A C(T, E, F)$ is equipped with the $\theta$-relative topology, then the collection of all polygonal functions is dense.

Proof. Let $G \in A C(T, E, F), p \in I$ and $\epsilon>0$. There exist $G_{1}, \cdots$, $G_{n} \in A C(T, F)$ and $x_{i} \in E$ such that $\left\|\Sigma G_{i} x_{i}-G\right\|_{B V, p}<\epsilon / 2$. Each $G_{i}$ is of the form $G_{i}=\hat{\mu}_{i}, \mu_{i} \ll m_{F}$. By Darst [3], there are simple functions $s_{i}$ such that

$$
\sum\left\|\mu_{i}-\lambda_{i}\right\| p\left(x_{i}\right)<\epsilon / 2
$$


where $\lambda_{i}$ is defined on $A$ by $\lambda_{i}(B)=\int_{B} s_{i} d m_{F}$. Let $s=\Sigma s_{i} x_{i}$. Then for the polygonal function $p_{s}$ we have

$$
\begin{aligned}
\left\|\sum G_{i} x_{i}-p_{s}\right\|_{B V, p} & =\left\|\sum \mu_{i} x_{i}-v_{s}\right\| \leqslant \sum\left\|\mu_{i} x_{i}-v_{s_{i} x_{i}}\right\|_{p} \\
& \leqslant \sum\left\|\mu_{i}-\lambda_{i}\right\| p\left(x_{i}\right)<\epsilon / 2 .
\end{aligned}
$$

By the triangle inequality we have $\left\|G-p_{s}\right\|_{B V, p}<\epsilon$ which completes the proof.

We omit the proof of the following easily established lemma.

LEMMA 2. If $E$ is complete, then $(M(A, E), \omega)$ is complete.

THEOREM 3. Let $\hat{E}$ be the completion of $E$. Then the completion of the space $A C(T, E, F)$ under the relative $\theta$-topology is the space $A C(T, \hat{E}, F)$.

Proof. First of all we observe that $A C(T, \hat{E}, F)$ is complete as a closed subspace of the complete space $(B V(T, \hat{E}), \theta)$. For $p$ in $I$ let $\hat{p}$ denote the unique continuous extension of $p$ to $\hat{E}$. Let $G=\Sigma_{i=1}^{n} G_{i} x_{i}$, with $x_{i} \in \hat{E}$ and $G_{i} \ll F$, and let $\epsilon>0$ and $p \in I$. Since $E$ is dense in $\hat{E}$ there exist $y_{1}, \cdots, y_{n}$ in $E$ such that

$$
\sum\left\|G_{i}\right\|_{B V} \hat{p}\left(x_{i}-y_{i}\right)<\epsilon .
$$

If $H=\Sigma G_{i} y_{i}$, then $\|G-H\|_{B V, \hat{p}} \leqslant \Sigma\left\|G_{i}\right\|_{B V} \hat{p}\left(x_{i}-y_{i}\right)<\epsilon$. This shows that $G$ is in the closure of $A C(T, E, F)$ in $A C(T, \hat{E}, F)$. Since the set $\left\{\Sigma G_{i} s_{i}: G_{i} \in\right.$ $\left.A C(T, F), s_{i} \in \hat{E}\right\}$ is dense in $A C(T, \hat{E}, F)$, the result follows.

4. Representation of continuous operators on spaces of vector measures. By Theorem 1, the space $A C(T, E, F)$ can be identified with the space of all $E$ valued measures on $A$ which are absolutely continuous with respect to the positive measure $m_{F}$. Therefore, to describe continuous operators on $A C(T, E, F)$ it suffices to describe continuous operators on the space of measures which are absolutely continuous with respect to $m_{F}$. In this section we will study the problem more generally. $\left({ }^{1}\right)$ Let $\Sigma$ be an algebra of subsets of a set $X$. Denote by $M(\Sigma, E)$ the space of all $E$-valued measures $m$ on $\Sigma$ such that for each $p \in I$ we have $\|m\|_{p}=\sup \Sigma p\left(m\left(F_{i}\right)\right)<\infty$ where the supremum is taken over the class of all finite partitions $\left\{F_{i}\right\}$ of $X$ into sets in $\Sigma$. Let $\omega$ denote the locally convex topology on $M(\Sigma, E)$ generated by the family of seminorms $\left\{\|\cdot\|_{p}: p \in \Gamma\right.$. Let $\mu \neq 0$ a fixed nonnegative finitely additive measure on $\Sigma$ and let $A C(\Sigma, E, \mu)$ denote the $\omega$-closure in $M(\Sigma, E)$ of the space spanned by the class of all measures of the form $\lambda x$ where $x \in E$ and $\lambda$ runs through the family of all bounded real-

(1) The author wishes to thank the referee for suggesting that he look into the problem in this general form. 
valued measures on $\Sigma$ which are absolutely continuous with respect to $\mu$. Let $\tau$ be the relative $\omega$-topology on $A C(\Sigma, E, \mu)$. We will represent the continuous linear operators from $(A C(\Sigma, E, \mu), \tau)$ into a locally convex space $H$.

For $s$ an $E$-valued simple function, we define $m_{s}: \Sigma \rightarrow E$ by $m_{s}(F)=$ $\int_{F} s d \mu$. It is clear that $m_{s} \in A C(\Sigma, E, \mu)$.

LEMma 3. The class of all measures of the form $m_{s}$ is $\tau$-dense in $A C(\Sigma, E, \mu)$.

Proof. Let $m \in A C(\Sigma, E, \mu), p \in I$ and $\epsilon>0$. By definition there are real-valued measures $\mu_{1}, \cdots, \mu_{n}$, which are absolutely continuous with respect to $\mu$, and $x_{i} \in E$ such that $\left\|\Sigma \mu_{i} x_{i}-m\right\|_{p}<\epsilon / 2$. By Darst [3] there are real-valued simple functions $s_{i}$ such that $\Sigma\left\|\mu_{i}-\lambda_{i}\right\| p\left(x_{i}\right)<\epsilon / 2$ where $\lambda_{i}$ is defined on $\Sigma$ by $\lambda_{i}(B)=\int_{B} s_{i} d \mu$. Let $s=\Sigma s_{i} x_{i}$. Then

$$
\left\|m_{s}-m\right\|_{p} \leqslant\left\|m-\sum \mu_{i} x_{i}\right\|_{p}+\sum\left\|\lambda_{i}-\mu_{i}\right\| p\left(x_{i}\right)<\epsilon .
$$

The lemma is proved.

Let now $H$ be another real locally convex space. We will represent the continuous linear operators from $(A C(\Sigma, E, \mu), \tau)$ into $H$.

Definition. Let $K$ be a function on $\Sigma$ with values in the space $L(E, H)$ of all linear operators from $E$ into $H$. Then $K$ is called convex relative to $\mu$ if, whenever $\left\{B_{j}\right\}$ is a finite partition of $B$ into sets in $\Sigma$, then $K(B)=\Sigma \alpha_{j} K\left(B_{j}\right)$ where $\alpha_{j}=\mu\left(B_{j}\right) / \mu(B)$. We take $0 / 0=0$. According to this convention it follows that $K(B)=0$ whenever $\mu(B)=0$. The function $K$ is called bounded if, for each continuous seminorm $q$ on $H$, there exists $p \in I$ such that

$$
\sup q(K(B) x)=\|K\|_{p, q}<\infty
$$

where the supremum is taken over the family of all sets $B$ in $\Sigma$ and all $x \in E$ with $p(x) \leqslant 1$.

Definition. Let $K$ be a convex (relative to $\mu$ ) bounded $L(E, H)$-valued function on $\Sigma$. The $v$-integral with respect to $K$ of an $E$-valued measure $m$ on $\Sigma$ is defined to be the $\lim \Sigma K\left(B_{i}\right) m\left(B_{i}\right)$, when it exists, where the limit is taken over the collection of all finite $\Sigma$-partitions (i.e., partitions into members of $\Sigma$ ) $\left\{B_{i}\right\}$ of $X$. In this case we say that $m$ is $v$-integrable with respect to $K$ and we denote the integral by $v \int m d K$.

We omit the proof of the following easily established lemma.

Lemma 4. Let $K$ be convex and bounded and let $s$ be an E-valued simple function. Then $m_{s}$ is v-integrable. Moreover there exists a finite $\Sigma$-partition $\left\{B_{i}\right\}$ of $X$ such that 


$$
v \int m_{s} d K=\sum K\left(F_{j}\right) m_{s}\left(F_{j}\right)
$$

for every finite $\Sigma$-partition $\left\{F_{j}\right\}$ of $X$ which is a refinement of $\left\{B_{i}\right\}$.

Lemma 5. Let $K$ be convex and bounded and assume that $H$ is complete. Then:

(1) Every $m$ in $A C(\Sigma, E, \mu)$ is v-integrable with respect to $K$.

(2) The map $m \rightarrow \phi_{K}(m)=v \int m d K$, from $(A C(\Sigma, E, \mu), \tau)$ into $H$, is a continuous linear operator. Furthermore, if $\|K\|_{p, q}<\infty$, then

$$
\|K\|_{p, q}=\sup \left\{q\left(\phi_{K}(m)\right): m \in A C(\Sigma, E, \mu),\|m\|_{p} \leqslant 1\right\} .
$$

Proof. First of all we observe that, if $m$ is $v$-integrable and if $\|K\|_{p, q}<$ $\infty$, then

$$
q\left(\phi_{K}(m)\right) \leqslant\|K\|_{p, q}\|m\|_{p} .
$$

Let now $m \in A C(\Sigma, E, \mu), q$ a continuous seminorm on $H$ and $\epsilon>0$. Let $p \in I$ be such that $\|K\|_{p, q}=d<\infty$. Let $s$ be an $E$-valued simple function such that $\left\|m_{s}-m\right\|_{p}<\epsilon / 2 d$.

By Lemma 4, there exists a finite $\Sigma$-partition $\left\{B_{i}\right\}$ of $X$ such that $\phi_{K}\left(m_{s}\right)=$ $\Sigma K\left(F_{j}\right) m_{s}\left(F_{j}\right)$ for each finite $\Sigma$-partition $\left\{F_{j}\right\}$ of $X$ which is a refinement of $\left\{B_{i}\right\}$. Now if $\left\{F_{j}\right\}$ and $\left\{G_{l}\right\}$ are both $\Sigma$-partitions of $X$ which are refinements of $\left\{B_{i}\right\}$, then

$$
\begin{aligned}
& q\left(\sum K\left(F_{j}\right) m\left(F_{j}\right)-\sum K\left(G_{l}\right) m\left(G_{l}\right)\right) \\
& \quad \leqslant q\left(\sum K\left(F_{j}\right) m\left(F_{j}\right)-\sum K\left(F_{j}\right) m_{s}\left(F_{j}\right)\right)+q\left(\sum K\left(G_{l}\right) m_{s}\left(G_{l}\right)-\sum K\left(G_{l}\right) m\left(G_{l}\right)\right) \\
& \quad \leqslant d\left\|m-m_{s}\right\|_{p}+d\left\|_{m}-m_{s}\right\|_{p}<\epsilon .
\end{aligned}
$$

This shows that the net $\left\{\Sigma K\left(F_{j}\right) m\left(F_{j}\right)\right\}$ is a Cauchy net in $H$ and hence convergent. This proves (1).

The inequality (*) implies that $\phi_{K}$ is a continuous linear map and that

$$
\|K\|_{p, q} \geqslant\left\|\phi_{K}\right\|_{p, q}=\sup \left\{q\left(\phi_{K}(m)\right): m \ll \mu,\|m\|_{p} \leqslant 1\right\} .
$$

Let now $\epsilon>0$ be given. There exists $F \in \Sigma$ and $x \in E$, with $p(x) \leqslant 1$, such that

$$
q(K(F) x)>\|K\|_{p, q}-\epsilon=a .
$$

Let $s=\chi_{F} x$. Then $\left\|m_{s}\right\|_{p}=p(x) \mu(F) \leqslant \mu(F)$. Also $K(F) m_{s}(F)=\mu(F) K(F) x$.

Thus

$$
q\left(\phi_{K}\left(m_{s}\right)\right)=\mu(F) q(K(F) x) \geqslant a \mu(F) .
$$


Hence $\left\|\phi_{K}\right\|_{p, q} \geqslant a=\|K\|_{p, q}-\epsilon$. Since $\epsilon>0$ was arbitrary, the result follows.

LEMMA 6. If $\phi$ is a continuous linear operator from $A C(\Sigma, E, \mu)$ into $H$, then there exists a unique convex bounded function $K$ such that $\phi=\phi_{K}$.

Proof. For each $F$ in $\Sigma$, let $\lambda_{F}$ be defined on $\Sigma$ by

$$
\lambda_{F}(B)=\mu(B \cap F) / \mu(F) .
$$

Clearly $\lambda_{F}$ is a bounded real-valued measure on $\Sigma$ and $\lambda_{F} \leqslant \mu$. We define $K$ on $\Sigma$ by

$$
K(F) x=\phi\left(\lambda_{F} x\right), \quad x \in E .
$$

Then $K$ is an $L(E, H)$-valued function. It is easy to see that $K$ is convex relative to $\mu$. Also $K$ is bounded. In fact, let $q$ be a continuous seminorm on $H$. Since $\phi$ is continuous there exist $p \in I$ and $M>0$ such that $q(\phi(m)) \leqslant M$ whenever $\|m\|_{p} \leqslant 1$. Thus $q(K(B) x) \leqslant M$ whenever $p(x) \leqslant 1$ since $\left\|\lambda_{B} x\right\|_{p} \leqslant 1$ whenever $p(x) \leqslant 1$. This proves that $K$ is bounded. Next we show that $\phi=\phi_{K}$. To this end, it suffices, by Lemma 3, to show that $\phi\left(m_{s}\right)=\phi_{K}\left(m_{s}\right)$ for each $E$-valued simple function $s$. Since $m_{s_{1}+s_{2}}=m_{s_{1}}+m_{s_{2}}$, it suffices to prove the claim for any $s$ of the form $s=\chi_{F} x, F \in \Sigma, x \in E$. But, for $s=\chi_{F} x$, we have $m_{s}=\mu(F) \lambda_{F} x$. Thus

$$
\phi\left(m_{s}\right)=\mu(F) \phi\left(\lambda_{F} x\right)=\mu(F) K(F) x=K(F) m_{s}(F)=\phi_{K}\left(m_{s}\right) .
$$

It follows that $\phi=\phi_{K}$. Finally, assume that $K_{1}$ is another convex bounded function such that $\phi=\phi_{K_{1}}$. If $K_{2}=K-K_{1}$, then $\phi_{K_{2}}=0$. We claim that $K_{2}=0$. Assume the contrary and let $F \in \Sigma$ be such that $K_{2}(F) \neq 0$. Choose $x \in E$, with $K_{2}(F) x \neq 0$, and set $s=\chi_{F} x$. Then $0=\phi_{K_{2}}\left(m_{s}\right)=\mu(F) K_{2}(F) x \neq$ 0 since $\mu(F) \neq 0$. This contradiction completes the proof.

Combining the preceding lemmas we get the following representation theorem.

THEOREM 4. If $H$ is complete, then the map $K \rightarrow \Phi_{K}$ is a one-to-one linear map from the space of all convex (relative to $\mu$ ) bounded $L(E, H)$-valued functions onto the space of all continuous linear operators from $A C(\Sigma, E, \mu)$ into $H$.

\section{BIBLIOGRAPHY}

1. Richard A. Alò and André de Korvin, Functions of bounded variation on idempotent semigroups, Math. Ann. 194 (1971), 1-11. MR 45 \#2454.

2. R. A. Alò, André de Korvin and R. J. Easton, Vector valued absolutely continuous functions on idempotent semigroups, Trans. Amer. Math. Soc. 172 (1972), 491-500. MR 46 \#9283.

3. R. B. Darst, A decomposition of finitely-additive set functions, J. Reine Angew. Math. 210 (1962), 31-37. MR 25 \#1257. 
4. N. Dinculeanu, Vector measures, Hochschulbücher fur Math. Bd. 64, VEB Deutscher Verlag, Berlin, 1966. MR 34 \#6011a.

5. N. Dunford and J. T. Schwartz, Linear operators. I: General theory, Pure and Appl. Math., vol. 7, Interscience, New York, 1958. MR 22 \#8302.

6. J. R. Edwards and S. G. Wayment, Representations for transformations continuous in the BV norm, Trans. Amer. Math. Soc. 154 (1971), 251-265. MR 43 \#466.

7. T. H. Hildebrandt, Linear continuous functionals on the space $(B V)$ with weak topologies, Proc. Amer. Math. Soc. 17 (1966), 658-664. MR 33 \#1710.

8. S. E. Newman, Measure algebras and functions of bounded variation on idempotent semigroups, Trans, Amer. Math. Soc. 163 (1972), 189-205. MR 46 \#7800.

9. - Measure algebras and functions of bounded variation on idempotent semigroups, Bull. Amer. Math. Soc. 75 (1969), 1396-1400. MR 40 \#4778.

DEPARTMENT OF MATHEMATICS, SOUTHERN ILLINOIS UNIVERSITY, CARBONDALE, ILLINOIS 62901

Current address: Instituto de Matemática, Universidade Estadual de Campinas, Caixa Postal 1170, 13100 Campinas, São Paulo, Brazil 